\title{
Obstacle Detector at Head Level
}

\section{Detector de obstáculos a nivel de cabeza}

\author{
V. Hernández Arreola ${ }^{1 *}$, F. J. Renero Carrillo ${ }^{1}$ \\ 1. Instituto Nacional de Astrofísica, Óptica y Electrónica (INAOE) \\ Puebla, México \\ (*) E-mail: veronica.hdez.a@gmail.com
}

Received: $15 / 08 / 2019 \quad$ Accepted: 26/11/2019

DOI: $10.7149 /$ OPA.52.4.51028

\begin{abstract}
:
In this paper, the design, manufacturing process and testing of a device able to detect obstacles at head level is presented. The device's functionality is based on emission and detection of infrared light and the tactile stimulus. The design was performed in order to detect objects, from the head and up to 150 $\mathrm{cm}$ away. The fabrication was done using both an infrared emitter and detector with a $940 \mathrm{~nm}$ wavelength; for the signals processing a commercial LM567 Texas Instruments $₫$ chip was used. Tests were performed on closed and open spaces, obtaining tactile response with objects placed at $150 \mathrm{~cm}$ on closed spaces and placed at $100 \mathrm{~cm}$ in open spaces. To further improve the device's performance on open spaces an optical filter was used on the phototransistor.
\end{abstract}

Key words: Obstacle's detection, Blind, Visual disability, Infrared emission and detection.

\section{RESUMEN:}

En este trabajo, se muestra el diseño, fabricación y pruebas de un dispositivo capaz de detectar obstáculos al nivel de la cabeza. El funcionamiento del dispositivo se basa en la emisión y detección de luz infrarroja y la estimulación táctil. El diseño se realizó para detectar objetos desde la cabeza y hasta $150 \mathrm{~cm}$ de distancia. La fabricación se realizó utilizando un emisor y un detector de infrarrojo con una longitud de onda de $940 \mathrm{~nm}$; para el procesamiento de las señales se utilizó un chip comercial LM567 de Texas Instruments ${ }^{\circledR}$. Las pruebas se realizaron en espacios cerrados y abiertos, obteniendo respuesta táctil con objetos a $150 \mathrm{~cm}$ en espacios cerrados y a $100 \mathrm{~cm}$ en espacios abiertos. Para utilizar el dispositivo en espacios abiertos, se colocó un filtro óptico en el fototransistor.

Palabras clave: Detección de obstáculos, ceguera, discapacidad visual, emisión y detección de infrarrojo.

\section{REFERENCES AND LINKS / REFERENCIAS Y ENLACES}

[1] Organización Mundial de la Salud OMS, "Ceguera y discapacidad visual", (2018).

[2] S. Hoefer, "Meet The Tacit Project. Its Sonar For The Blind", Grathio Labs, 8, (2011).

[3] C. Sánchez, "El futuro del bastón blanco: tecnología inteligente por y para ciegos", (2018).

[4] W. Martin, K. Dancer, K. Rock, C. Zeleny, K. Yelamarthi, "The smart cane: An electrical engineering design project", In ASEE North Central Section Conference. (2009).

[5] F. A. Reyes Moreno, "Diseño y construcción de un dispositivo electrónico de ayuda y entretenimiento para personas con discapacidad visual a través de ondas vibratorias e interfaces audibles para el proyecto HANDEYES del banco de ideas del SENESCYT", Bachelor's thesis, ESPE. (2016).

[6] Instituto de Tecnologías Educativas, “Educación Inclusiva: Discapacidad Visual”,(2018)

[7] E. W. Hill, P. Ponder, "Orientation and mobility techniques: A guide for the practitioner", Amer Foundation for the Blind. (1976). ISBN: 0891280014. 
[8] A. F. Villan, J. L. Candas, R. U. Fernandez, R. C. Tejedor, "Face recognition and spoofing detection system adapted to visually-impaired people", IEEE Latin America Transactions, 14, (II), 913-921. (2016).

\section{Introducción}

En el mundo hay aproximadamente 1300 millones de personas con discapacidad visual, de las cuales 36 millones son ciegas y 1264 millones son débiles visuales [1]. Las personas con deficiencia visual (P.D.V.), tienen problemas para realizar sus actividades diarias fuera de su zona de confort, debido en gran medida a los obstáculos que encuentran en sus trayectos. La sociedad provee a las P.D.V. de ayudas para reducir los riesgos de lesiones, tales como ranuras-guías para el bastón en las banquetas, guías audibles en los cruces de calles y sistemas de transporte, entre otras.

Así las P.D.V. pueden auxiliarse con el bastón y su capacidad auditiva, al igual que con un perro guía, para poder localizar obstáculos o relieves a nivel del suelo, sin embargo no proporciona información sobre el resto de su entorno, ya que no cuentan con algún dispositivo que les informe de la presencia de obstáculos localizados por encima del nivel de suelo, por lo cual, desde la mano que sujeta el bastón, y hasta la cabeza son partes del cuerpo que están expuestas a colisiones.

En la Fig. 1 se muestran ejemplos de objetos localizados en la vía pública que no pueden ser detectados por las P.D.V. mediante el bastón o el perro guía, y que pueden llegar a provocarles lesiones.
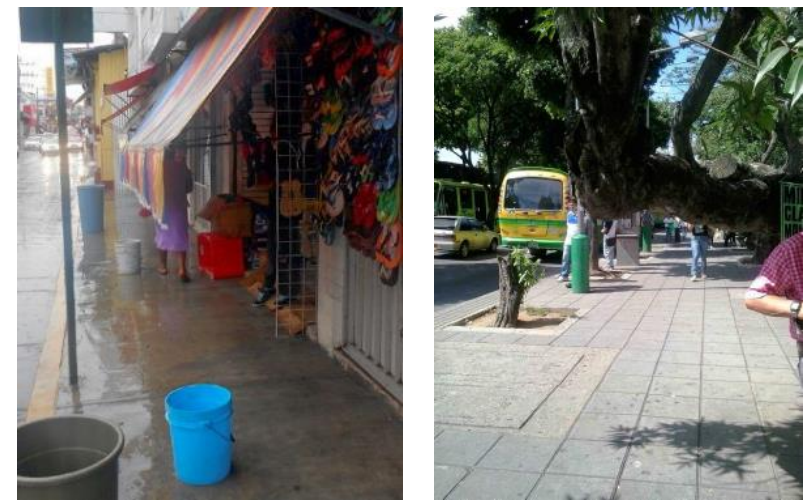

Fig. 1. Fotografías de obstáculos encontrados en los trayectos de los transeúntes. Estos obstáculos son difíciles de detectar para las PDV utilizando el bastón blanco o perro guía.

En este trabajo se presenta un Dispositivo para la Detección de Obstáculos a Nivel de Cabeza (D.O.N.C), está basado en la emisión y detección de luz infrarroja. La luz infrarroja es irradiada por el emisor en la dirección de desplazamiento, y al ser reflejada por el obstáculo, es detectada por el fototransistor. La corriente producida por la detección es transmitida al individuo mediante estimulación táctil, y es proporcional a la cercanía del obstáculo.

El D.O.N.C. se diseñó para detectar obstáculos desde la cabeza, ubicación del emisor de luz infrarroja, y hasta $150 \mathrm{~cm}$ de distancia al obstáculo. Las pruebas en espacios cerrados demostraron un funcionamiento de acuerdo a lo esperado en el diseño, mientras que en espacios abiertos, debido a la presencia de la radiación solar, la distancia de detección se ve reducida a un $70 \%$.

\section{Antecedentes}

A lo largo de la historia se han desarrollado diversos dispositivos para facilitar la movilidad de las personas con discapacidad visual, el ejemplo más antiguo de ello es el bastón blanco, inventado en 1930 como un dispositivo auxiliar para permitir el desplazamiento con una mayor autonomía, además de servir como distintivo y como protección. Actualmente es el dispositivo más utilizado en todo el mundo, razón por la cual en los últimos años se han hecho desarrollos para crear un bastón blanco inteligente, que permita a las P.D.V. localizar los obstáculos a su alrededor y tomar una decisión al respecto. Existen diversos dispositivos 
diseñados, desde sensores sencillos que emiten un sonido para indicar sobre la presencia de obstáculos, hasta cámaras y procesadores que describen el entorno que rodea al usuario.

Algunos dispositivos tales como TACIT [2] están diseñados como un complemento a los dispositivos de asistencia, o como EGARA [3] y SMARTCANE [4], son una mejora del bastón blanco que permite detectar objetos a una mayor altura, o como HANDEYES [5] que permiten colocar el dispositivo en diversos objetos para detección a diferentes alturas. Estos dispositivos utilizan el sentido del oído de las P.D.V. para alertarlas sobre los obstáculos en sus trayectos.

Algo indispensable en cada uno de estos desarrollos es la instrucción y adaptación, que permite a las P.D.V. adaptarse a esta nueva forma de "ver", ya sea mediante un bastón con sonido característico u otro tipo de estímulo $[6,7,8]$.

\section{Detección de obstáculos por radiación infrarroja}

La mayoría de los detectores de obstáculos están basados en la emisión y detección de algún tipo de señal. Entre una de las más empleadas son las basadas en radiación electromagnética, que van desde luz visible hasta ultravioleta, rayos $\mathrm{X}$, infrarrojo, entre otros.

La radiación infrarroja es una fracción de la radiación electromagnética de mayor longitud de onda que el visible $(800 \mathrm{~nm}$ a $1 \mathrm{~mm})$. Para la detección de objetos se requiere de una fuente de emisión de radiación infrarroja, LED IR (Light-Emitting Diode), y un sensor que colecte la radiación reflejada o transmitida por el objeto. Actualmente existen diversos dispositivos que permiten realizar el censado de la radiación infrarroja, como son los fotodiodos y los fototransistores que varían sus propiedades en función de la radiación infrarroja detectada.

Algunos de los arreglos para la emisión y detección de la radiación infrarroja son:

-De barrera, en los cuales el fotodetector se coloca frente al emisor IR, de forma que al encontrarse un objeto entre ellos se limite la detección y cambie la respuesta del fotodetector.

-De reflexión, en los cuales el fotodetector y el emisor IR se colocan en paralelo, con lo cual al encontrarse un objeto reflexivo frente a ellos permita que el fotodetector reciba la radiación infrarroja y cambie su respuesta.

En la Fig. 2 se muestra el esquema del D.O.N.C. Está compuesto de dos elementos ópticos, un LED IR y un fototransistor IR, y de un motor vibrador. El dispositivo está basado en un arreglo de reflexión, la emisión de radiación IR a través del LED IR es reflejada por el objeto y detectada por el fototransistor, para activar el motor vibrador mediante una corriente proporcional a la distancia del objeto detectado.

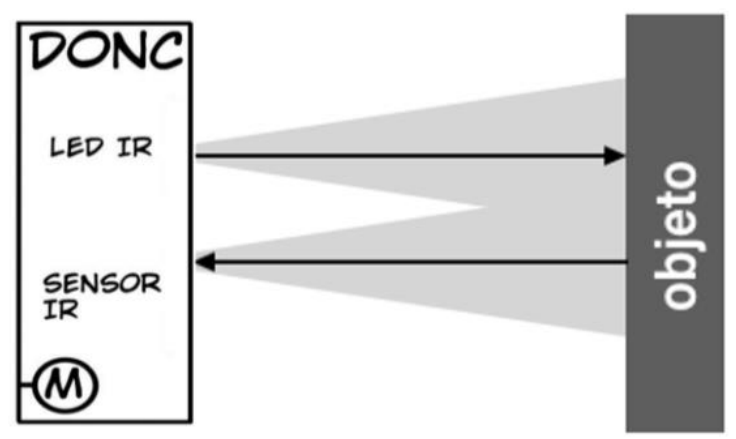

Fig. 2. Esquematización del funcionamiento del D.O.N.C. El LED IR emite la radiación IR, que es reflejada por el obstáculo, y registrada por el sensor IR, LED IR en la parte superior y fototransistor en la parte inferior. El circuito electrónico activa el LED IR y procesa la señal del sensor, para transmitir la señal al micro-motor vibrador. 


\section{Diseño del D.O.N.C.}

\section{4.a. Diseño óptico}

En la figura 3 se muestra el esquema para el diseño óptico geométrico del D.O.N.C. En este esquema $\theta$ representa el ángulo para la máxima radiación emitida por el LED IR, y $\rho$ el ángulo de campo del sensor IR. A, B y C representa la separación entre LED IR y el sensor IR, distancia del LED al objeto, y C porción del objeto que refleja la radiación IR.

Para radiar mayor área posible implica alejar al objeto, es decir la distancia B tan grande como sea posible. Además, en esta geometría, de acuerdo con la ley de la reflexión de las ondas electromagnéticas y para una superficie plana, para colectar la mayor cantidad de radiación reflectado por el objeto (distancia C), la distancia A sería cercana a cero.

El LED IR se consideró cómo una fuente de radiación constante que no cambia con la distancia, sin embargo, considerando las características reales de los componentes, el LED IR se comporta como una fuente de radiación Lambertiana, i.e., la intensidad de radiación decrece como el inverso al cuadrado de la distancia al objeto. Adicionalmente, se supone el comportamiento de la superficie del objeto detectado como una superficie isotrópica, con lo cual el fototransistor detectara la radiación proveniente de una fuente Lambertiana. Por lo cual, la intensidad de radiación del LED IR y la sensibilidad de detección del fototransistor limitan la distancia de detección.

Así, basado en el diseño óptico, fuentes y fototransistores de IR comerciales, en la tabla I se muestra los parámetros para la detección de un obstáculo a 150 centímetros de distancia (B).

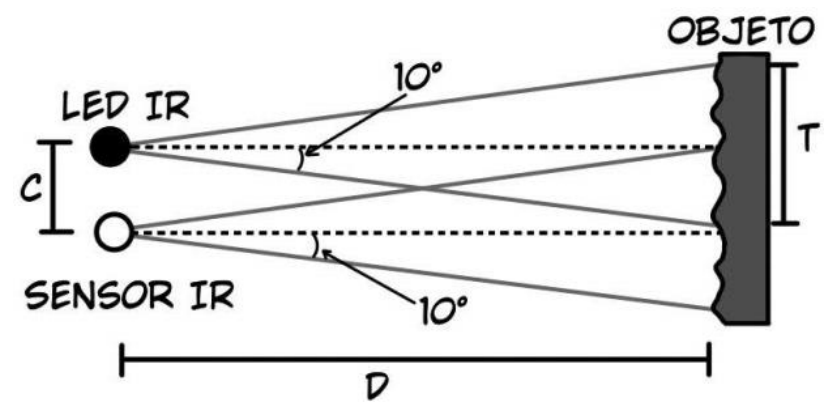

Fig. 3. Ilustración del diseño óptico del D.O.N.C. $\theta$ representa el ángulo para la máxima radiación emitida por el LED IR, y $\rho$ el ángulo de campo del sensor IR. $C$, $D$ y $T$ representa la separación entre LED y sensor IR, distancia del LED y sensor al objeto, y porción del objeto que refleja la radiación IR

Para el diseño, el LED IR se consideró como una fuente de radiación constante que no cambia con la distancia, sin embargo, considerando las características reales de los componentes, el LED IR se comporta como una fuente de radiación Lambertiana, i.e., la intensidad de radiación decrece como el inverso al cuadrado de la distancia al objeto. Adicionalmente, se supone el comportamiento de la superficie del objeto detectado como una superficie isotrópica, con lo cual el fototransistor detectara la radiación proveniente de una fuente Lambertiana. Por lo cual, la intensidad de radiación del LED IR y la sensibilidad de detección del fototransistor limitan la distancia a la que es posible detectar un objeto. La tabla I muestra los valores considerados para el diseño óptico. Considerando los valores de intensidad de emisión del LED IR comercial de $940 \mathrm{~nm}$ y la intensidad mínima requerida para la activación del fototransistor, así como el ángulo de emisión mínimo de $1^{\circ}$, se obtuvo la distancia entre el LED IR y el sensor IR a partir de la ecuación 1.

$$
\mathrm{C}=2 \mathrm{Dtan} 1^{\circ}=5.2 \mathrm{~cm}
$$

Con lo cual, considerando el LED IR como una fuente Lambertiana, la región del objeto que es radiado por el LED IR a $150 \mathrm{~cm}$ del D.O.N.C. es de $14 \mathrm{~cm}$. 
TABLA I

VALORES CALCULADOS CON EL DISEÑO ÓPTICO

\begin{tabular}{ccc}
\hline \hline Parámetro & Parámetro de diseño & Valor \\
\hline C & Distancia entre el LED IR y el Sensor IR & $5 \mathrm{~cm}$ \\
$\mathrm{D}$ & Distancia del LED IR al objeto & $150 \mathrm{~cm}$ \\
$\mathrm{~T}$ & Tamaño del objeto & $14 \mathrm{~cm}$ \\
$\mathrm{Ie}$ & Intensidad de emisión del LED IR (IR333-A) & $20 \mathrm{~mW} / \mathrm{sr}$ \\
Ia & Intensidad mínima de activación del & $0.5 \mathrm{~mW} / \mathrm{sr}$ \\
\hline \hline
\end{tabular}

\section{4.b. Diseño electrónico}

Para convertir la señal del fototransistor a una señal vibratoria, se implementó un circuito electrónico basado en la detección de fase, mediante el circuito integrado LM567 de TI@.

En la Fig. 4 se muestra el diseño electrónico del D.O.N.C. El circuito está basado en un decodificador de tono LM567, el cual está configurado como un oscilador controlado por tensión (VCO). Por medio del VCO se genera una señal en corriente de $25 \mathrm{~mA}$ con una frecuencia fvco $=5 \mathrm{KHz}$ (calculada mediante la ecuación 2) que es emitida a través del LED IR, controlado a partir del transistor 2N2222. Para la detección el filtro pasabanda es sintonizado a la misma frecuencia para que al detectar con el fototransistor la señal infrarroja reflejada por un objeto, la salida del decodificador de tono active el motor vibrador (controlado con el transistor BC558) con una corriente proporcional a la distancia del objeto. Si el objeto se encuentra cercano al D.O.N.C. la corriente será mayor y en caso de no detectar ningún objeto se mantendrá desactivado.

$$
\mathrm{f}_{V C O}=\frac{1.1}{R_{1} C_{1}}=\frac{1.1}{(10 k \Omega)(22 n F)}=5 \mathrm{KHz}
$$

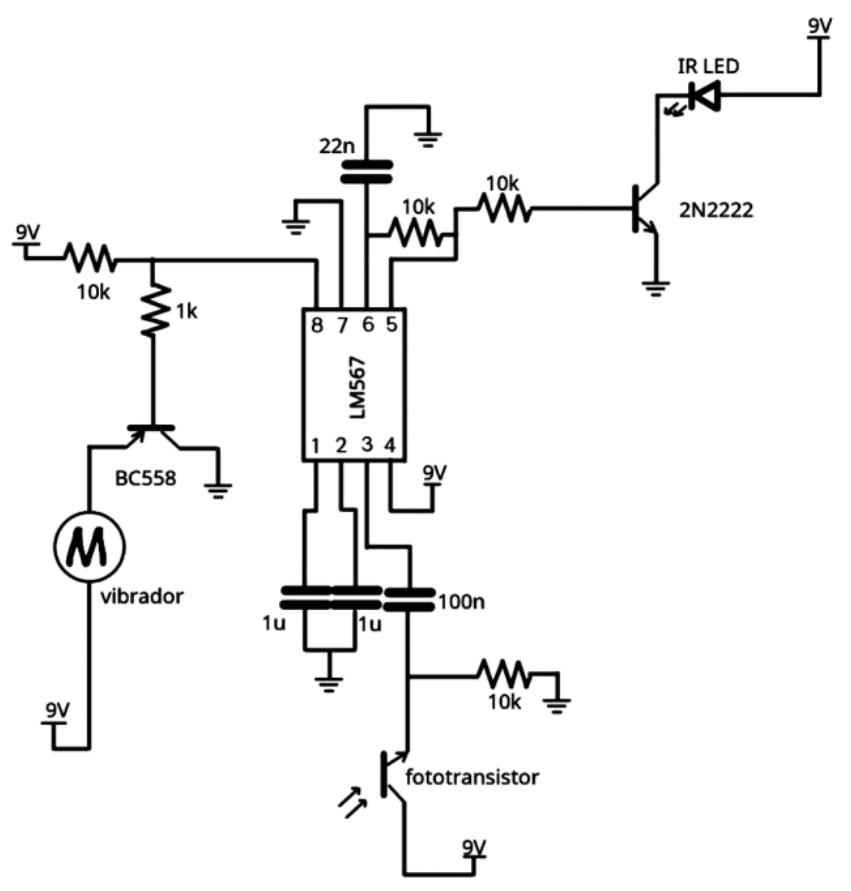

Fig. 4. Circuito electrónico del D.O.N.C. 


\section{Pruebas y resultados del D.O.N.C.}

Se realizó la caracterización estática del D.O.N.C. colocando objetos de diferentes materiales y dimensiones a una distancia de $150 \mathrm{~cm}$ sobre una mesa, obteniendo una respuesta del motor vibrador con los objetos de plástico, madera, PVC y aluminio en las tres dimensiones consideradas, como se puede observar en la tabla II.

Posteriormente se realizaron las pruebas en espacios cerrados, para controlar la radiación infrarroja proveniente del Sol, donde se colocaron los objetos utilizados para la caracterización estática a la altura de la cabeza de un voluntario (sin problemas visuales, que usó un antifaz para simular un individuo con ceguera). Al voluntario se le pidió caminar en dirección recta, al percibir la primer vibración disminuir la velocidad de marcha, y detenerse si la vibración aumentaba (la Fig. 5 se muestra el esquema de uso del D.O.N.C. y una fotografía del voluntario realizando la prueba). El voluntario pudo evitar la colisión con obstáculos de plástico, madera, PVC y aluminio.

De manera similar se realizaron pruebas en espacios abiertos, debido a la radiación solar fue colocado un filtro óptico para reducir el rango de radiación detectada por el fototransistor. Debido a la restricción de la radiación, la distancia de detección se redujo a un $70 \%$ y los objetos de menores dimensiones fueron detectados a una distancia de $100 \mathrm{~cm}$.

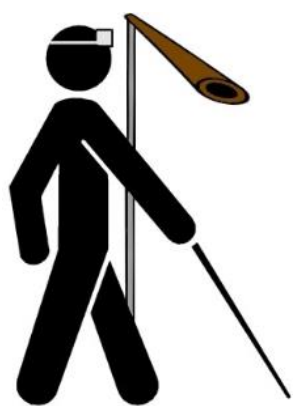

(a)

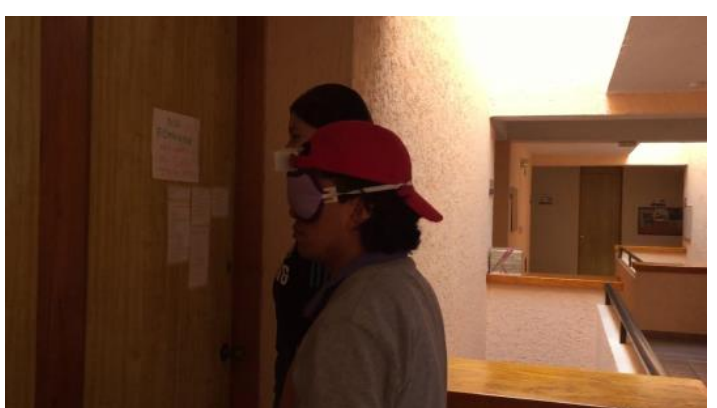

(b)

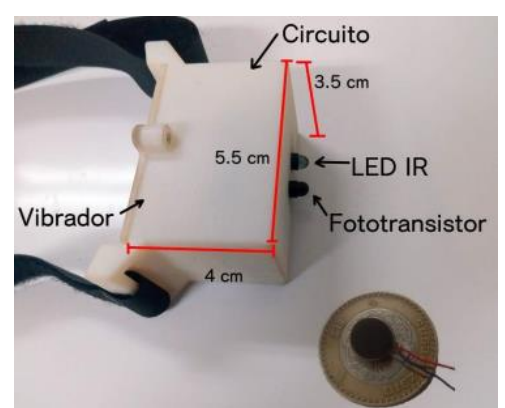

(c)

Fig. 5. a) Esquematización del D.O.N.C., b) pruebas en espacios cerrados del funcionamiento del D.O.N.C. y c) fotografía del D.O.N.C. y motor vibrador utilizado.

TABLA II

RESULTADOS DE DETECCIÓN

\begin{tabular}{ccc}
\hline \hline Material & Dimensiones & Detección \\
\hline \multirow{3}{*}{ Plástico } & $10 \mathrm{~cm} \times 5 \mathrm{~cm}$ & $\checkmark$ \\
& $25 \mathrm{~cm} \times 7 \mathrm{~cm}$ & $\checkmark$ \\
& $30 \mathrm{~cm} \times 10 \mathrm{~cm}$ & $\checkmark$ \\
\hline \multirow{3}{*}{ Madera } & $10 \mathrm{~cm} \times 5 \mathrm{~cm}$ & $\checkmark$ \\
& $25 \mathrm{~cm} \times 7 \mathrm{~cm}$ & $\checkmark$ \\
PVC & $30 \mathrm{~cm} \times 10 \mathrm{~cm}$ & $\checkmark$ \\
& $10 \mathrm{~cm} \times 5 \mathrm{~cm}$ & $\checkmark$ \\
& $25 \mathrm{~cm} \times 7 \mathrm{~cm}$ & $\checkmark$ \\
Vidrio & $30 \mathrm{~cm} \times 10 \mathrm{~cm}$ & $\checkmark$ \\
& $10 \mathrm{~cm} \times 5 \mathrm{~cm}$ & $\times$ \\
& $25 \mathrm{~cm} \times 7 \mathrm{~cm}$ & $\times$ \\
\multirow{2}{*}{ Aluminio } & $30 \mathrm{~cm} \times 10 \mathrm{~cm}$ & $\times$ \\
& $10 \mathrm{~cm} \times 5 \mathrm{~cm}$ & $\checkmark$ \\
& $25 \mathrm{~cm} \times 7 \mathrm{~cm}$ & $\checkmark$ \\
\hline \hline
\end{tabular}

Resultados de detección en pruebas con diferentes materiales y tamaños 


\section{Conclusiones}

El diseño basado en un emisor y un fototransistor de IR permite una correcta detección, en interiores, de los obstáculos localizados a la altura de la cabeza del usuario. A diferencia de otros dispositivos, el D.O.N.C. facilita la movilidad sin comprometer ningún sentido. La ubicación del D.O.N.C. y el sistema de estimulación facilitan al usuario la adaptación a su uso.

En las pruebas en espacios cerrados, el voluntario detecto correctamente los obstáculos de plástico, madera, PVC y aluminio a nivel de su cabeza a una distancia de $150 \mathrm{~cm}$. El D.O.N.C. está limitado a la reflexión de la radiación infrarroja del obstáculo. Además, en espacios abiertos se detectaron problemas debidos al amplio rango de detección del fototransistor, sin embargo es posible utilizar filtros ópticos que limiten su rango de detección a la longitud de onda emitida por el LED IR, con lo cual la distancia de detección se reduce a un $70 \%$.

El área cubierta por el D.O.N.C. en espacios cerrados es de $52.9 \mathrm{~cm}$ x $26.5 \mathrm{~cm}$ a una distancia de $150 \mathrm{~cm}$, lo que hace posible una correcta detección de objetos de plástico, madera, PVC y aluminio a la altura de la cabeza.

La inclinación de los objetos es determinante para su detección, haciendo posible solo la detección de objetos en los que la reflexión de la radiación infrarroja sea percibida por el fototransistor.

La detección de obstáculos a nivel de cabeza puede realizarse igualmente empleando sistemas de emisión y detección de señales, como es el caso de los detectores de ultrasonido, sin embargo se requiere un circuito más complejo para la detección de los objetos y por consiguiente de un costo mayor. Nuestro diseño es simple y económico para que cualquier persona con deficiencia visual pueda tenerlo.

\section{Acknowledgements}

Es importante agradecer enormemente al Dr. Guillermo Espinosa Flores-Verdad por su asesoría y gran ayuda para resolver los problemas del diseño electrónico y su apoyo en la revisión de este trabajo.

Verónica Hernández Arreola agradece a CONACYT por su apoyo en el financiamiento mediante la beca para el programa de doctorado con número 449733. 